\title{
INFLUÊNCIA DA PRÉ-CONCENTRAÇÃO NO ÍNDICE DE ABRASIVIDADE DE MINÉRIOS DE COBRE E DE ZINCO*
}

\author{
Larissa de Mores Peres ${ }^{1}$ \\ Camila Peres Massola \\ Mauricio Guimarães Bergerman ${ }^{3}$
}

\begin{abstract}
Resumo
A utilização da pré-concentração de minerais é um processo já conhecido e que gera ganhos significativos em termos de aumento de teor de alimentação das usinas. Tal processo, porém, gera produtos de granulometria fina que dificultam, ou mesmo impossibilitam, a avaliação da abrasividade destes pelo ensaio de Bond, método mais utilizado atualmente na indústria mineral. O ensaio LCPC se apresenta, então, como uma alternativa para avaliação da abrasividade de materiais que se encontram em uma granulometria mais fina. O objetivo principal do trabalho apresentado neste artigo é avaliar o índice de abrasividade de minérios que passaram por um processo de pré-concentração e tal avaliação foi realizada por meio do LCPC. Foi possível observar que, para a amostra de cobre, a préconcentração resultou em um concentrado de abrasividade menor que a alimentação, o que torna o processo vantajoso como uma etapa anterior ao beneficiamento propriamente dito. Já no caso do zinco, observou-se uma maior abrasividade no concentrado, o que sugere a concentração dos minerais abrasivos no concentrado, ao contrário do que seria esperado, que seria uma maior concentração de minerais mais abrasivos na ganga.
\end{abstract}

Palavras-chave: Abrasividade; LCPC; Pré-concentração.

\section{INFLUENCE OF PRE-CONCENTRATION ON THE ABRASION INDEX OF COPPER AND ZINC ORES}

\section{Abstract}

Pre-concentration is an already known process in the mineral industry that can result in an expressively increase of grades. This process, however, also generates fine products, whose particles sizes difficult or even unable to evaluate their abrasiveness by the Bond Abrasion Index. An alternative to evaluate the abrasiveness of fine materials is the LCPC method. The main objective of this work is to assess the influence of the abrasive index of ores that underwent a preconcentration process. For one of the samples, the pre-concentration provided a concentrate less abrasive than the feed, which makes the process advantageous as a step prior to the processing. On the other hand, the zinc ore sample resulted in a concentrate more abrasive than the feed, which suggests the concentration of abrasive minerals in the concentrate, contrary to what would normally be expected.

Keywords: Abrasiveness; LCPC; Pre-concentration

Eng. de Minas, estudante, Escola Politécnica da USP, São Paulo/SP, Brasil.

2 Eng. de Minas, doutora, pesquisadora, Laboratório de Processos Metalúrgicos, Instituto de Pesquisas Tecnológicas do Estado de São Paulo S/A, São Paulo/SP, Brasil.

3 Eng. de Minas, doutor, professor, Departamento de Engenharia de Minas e de Petróleo da Universidade de São Paulo, São Paulo/SP, Brasil. 


\section{INTRODUÇÃO}

A utilização da pré-concentração de minerais é um processo já conhecido e que gera ganhos significativos em termos de aumento de teor de alimentação das usinas - o que pode levar ao aumento das reservas, redução da massa a ser processada para se produzir uma mesma quantidade de metal e possibilidade de geração de um rejeito grosseiro, que possui uma destinação mais simples e econômica que os rejeitos finos, os quais necessitam de barragens de grandes dimensões [1]. No entanto, a implantação de circuitos de pré-concentração é onerosa e em muitos casos os custos de implantação dos mesmos não são compensados pelo ganho operacional esperado pelas avaliações realizadas a partir de dados laboratoriais e de plantas piloto. Deve-se ressaltar, porém, que em geral não se leva em conta nestes cálculos de viabilidade os ganhos que podem ser obtidos com a redução do Wi e do Ai do material que irá alimentar a usina, caso seja pré-concentrado, já que com esta operação uma grande quantidade de minerais de ganga, de maior competência em relação à moagem e altas taxas de desgaste dos revestimentos e corpos moedores das usinas, é eliminada.

No Brasil, a pré-concentração já foi utilizada na mina de fluorita da Nitroquímica, paralisada desde 2009, em Morro da Fumaça, SC [2] e é aplicada industrialmente na mina de Vanádio da Largo Resources, em Maracás, BA. Avaliações em escala laboratorial e piloto em depósitos brasileiros para diferentes minérios sulfetados de cobre, níquel, zinco e chumbo [1] [3] [4] [5] [6] [7] [8] ilustram ganhos significativos em termos de aumento de teor com altas recuperações metalúrgicas, possibilitando o descarte de aproximadamente $50 \%$ da massa como rejeito, que retém menos que $10 \%$ do metal total dos minérios que constituem a alimentação do ensaio.

O objetivo principal do trabalho apresentado neste artigo é avaliar a influência do índice de abrasividade de minérios que passaram por um processo de préconcentração. Porém, como nos ensaios de pré-concentração em laboratório é difícil obter a massa e granulometria requeridas pelo ensaio de Ai de Bond, atualmente o método mais utilizado na indústria mineral [9], e como muitas vezes existem diferenças de granulometria entre o concentrado e o rejeito da pré-concentração, devido, por exemplo, à estratificação no processo de jigagem, é importante também se pesquisar um método alternativo ao de Bond para tal avaliação.

Considerando a importância de se conhecer a abrasividade de um determinado mineral que entrará na planta de beneficiamento, para avaliar o desgaste nos equipamentos do processo, o ensaio LCPC se apresenta como uma alternativa para avaliação da abrasividade de materiais que se encontram em uma granulometria mais fina.

Enquanto o Ensaio de Abrasividade de Bond, descrito por Bergstrom [10], requer $1600 \mathrm{~g}$ de minério apresentando granulometria entre $3 / 4$ " e $1 / 2$ ", o ensaio LCPC é realizado com apenas $500 \mathrm{~g}$ de material com granulometria bem mais fina, entre 4 $\mathrm{mm}$ e $6,3 \mathrm{~mm}$. Este ensaio foi desenvolvido pelo Laboratoire Central des Ponts et Chaussées, na França, na década de 80. O Índice LCPC pode ser usado como uma medida tanto de abrasividade quanto da influência da granulometria do material e os procedimentos deste ensaio são definidos pela Norma Francesa P18-579 [11]. 
Além de ser vastamente utilizado na Europa, o motivo da escolha do método foi, principalmente, por exigir uma menor massa de material e com uma granulometria menor que a requerida pelo método de Bond, ambas restrições existentes neste método e citadas anteriormente como as principais limitações desta técnica para avaliar a abrasividade de minérios pré-concentrados. Além disso, a facilidade em encontrar um fornecedor que fabricasse o equipamento especificado pela Norma foi também um fator fundamental para a utilização do método LCPC para as análises apresentadas.

Apresenta-se, então, neste artigo, a avaliação da abrasividade de minérios que tenham passado por um processo de pré-concentração por meio do ensaio LCPC, a fim de se avaliar a influência de tais processos na abrasividade dos produtos gerados. Desta forma, espera-se fornecer mais subsídios à avaliação econômica de projetos de pré-concentração, permitindo assim uma avaliação mais fiel desta alternativa.

\section{MATERIAIS E MÉTODOS}

As amostras utilizadas para o desenvolvimento do projeto são apresentadas na Tabela 1, e já foram recebidas pré-concentradas pelo processo de jigagem.

Tabela 1. Amostras utilizadas

\begin{tabular}{ccc}
\hline ID & \multicolumn{2}{c}{ Amostra } \\
\hline 1 & Minério Sulfetado de Cobre & Alimentação \\
2 & & Concentrado \\
3 & & Rejeito \\
\hline 4 & Minério Silicatado de Zinco & Alimentação \\
5 & & Concentrado \\
6 & & Rejeito \\
\hline
\end{tabular}

As amostras foram inicialmente pesadas e então manuseadas a fim de obter a massa requerida pelo ensaio, $500 \mathrm{~g}$, na granulometria entre $6,3 \mathrm{~mm}$ e $4 \mathrm{~mm}$.

Para garantir a homogeneidade e representatividade das alíquotas a serem trabalhadas, toda a amostra inicial disponível de cada minério foi britada abaixo de $6,3 \mathrm{~mm}$ em britador de mandíbulas e britador de rolos. As amostras foram então homogeneizadas, utilizando o método da pilha prismática alongada. A partir desta pilha, foram retiradas as alíquotas para os ensaios de abrasividade e caracterização. A caracterização das amostras foi realizada por meio das técnicas de fluorescência de raios-X (FRX) para determinação da composição química em termos de porcentagens de óxidos, e de difração de raios-X (DRX) para determinação da assembleia mineralógica.

Como já citado, o ensaio de abrasividade LCPC foi desenvolvido na França, na década de 80 , e os procedimentos deste são definidos pela Norma Francesa P18579. O equipamento LCPC utilizado para avaliar a abrasividade dos materiais é mostrado na Figura 1. 


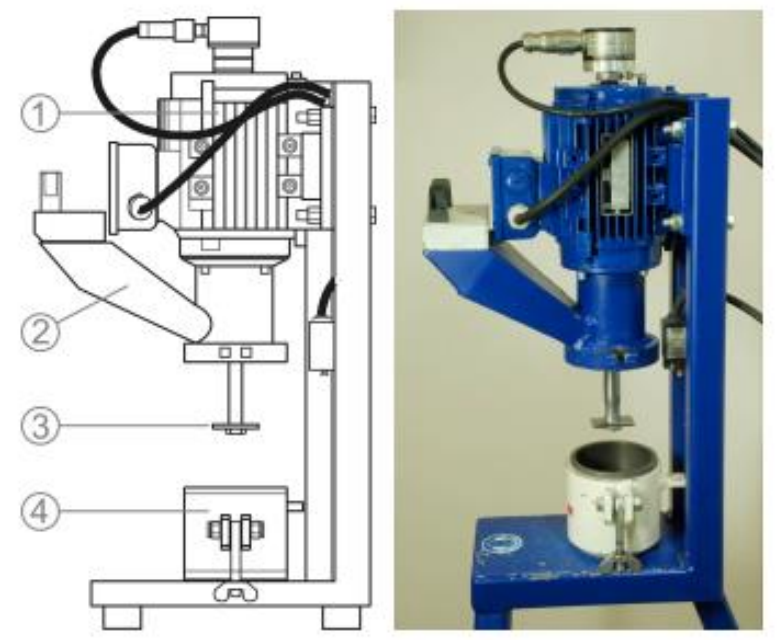

Figure 1. Equipamento LCPC (1-motor, 2-tubo de funil, 3-rotor e fixação da placa, 4-recipiente de amostra) [12].

O abrasímetro é composto por um motor que faz girar uma placa de metal dentro de um recipiente cilíndrico que contém $500 \mathrm{~g}$ de amostra com granulometria entre $6,3 \mathrm{~mm}$ e $4 \mathrm{~mm}$. A placa de metal padronizada deve ser fabricada em aço de baixo carbono (C1015), com dureza Rockwell em torno de 60 a 75 HRB e dimensões de $(50 \times 25 \times 5) \mathrm{mm}$.

O rotor, no qual a placa de metal está acoplada, gira por 5 minutos numa rotação de 4.500 rpm dentro do recipiente com a amostra. Para a determinação da abrasividade, a placa de metal é pesada antes e depois do teste e a perda de massa registrada é utilizada para o cálculo da abrasividade característica do material. Quanto maior a perda de massa determinada no ensaio, mais abrasivo será o material.

Segundo a Norma Francesa, o índice de abrasividade LCPC é calculado como mostrado na Equação 1:

$$
\mathrm{LCPC}=1000 \times 1000\left(\mathrm{~m}_{\mathrm{ip}}-\mathrm{m}_{\mathrm{fp}}\right) / \mathrm{M}
$$

Onde: $m_{\text {ip }}$ é a massa da plaquinha antes do teste LCPC;

$\mathrm{m}_{\mathrm{fp}}$ é a massa da plaquinha depois do teste LCPC; e

Mé a massa da amostra (500 $\pm 0,2 \mathrm{~g})$.

Para garantir que as placas utilizadas no ensaio apresentavam durezas corretas, realizaram-se medições e ensaios de dureza Rockwell $B$, antes de se iniciarem os ensaios propriamente ditos. A dureza média obtida para a placa do LCPC com os testes preliminares foi de $65 \mathrm{HRB}$, portanto dentro do intervalo de 60 a $75 \mathrm{HRB}$ definido pela Norma Francesa. Tomou-se o cuidado de usar placas do mesmo lote e do mesmo fabricante para realizar todos os ensaios, de modo a evitar que possíveis variações na fabricação dos lotes interferissem nos resultados do projeto. Além disso, para todas as amostras foram realizados testes em duplicata ou triplicata a fim de minimizar a influência de possíveis erros nos ensaios. 


\section{RESULTADOS E DISCUSSÃO}

As figuras 2 e 3 ilustram as amostras de minério (alimentação, concentração e rejeito da pré-concentração) utilizadas nesse trabalho, cujas caracterizações químicas e mineralógicas são apresentadas nas Tabelas 2 e 3 . A caracterização das amostras foi feita por meio dos métodos de difração de raio-x (DRX) e por absorção atômica (ICP).

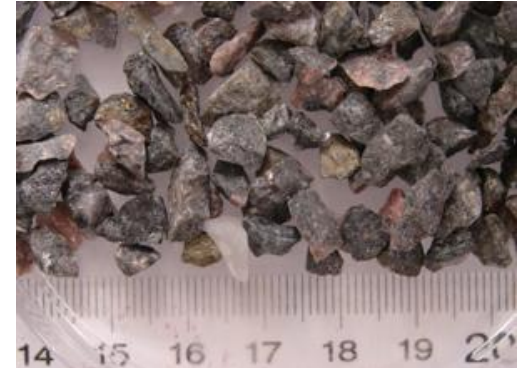

Figura 2. Amostra de minério sulfetado de cobre de $6,3 \mathrm{~mm}$ a $4 \mathrm{~mm}$. Da esquerda para direita, alimentação, concentrado e rejeito.
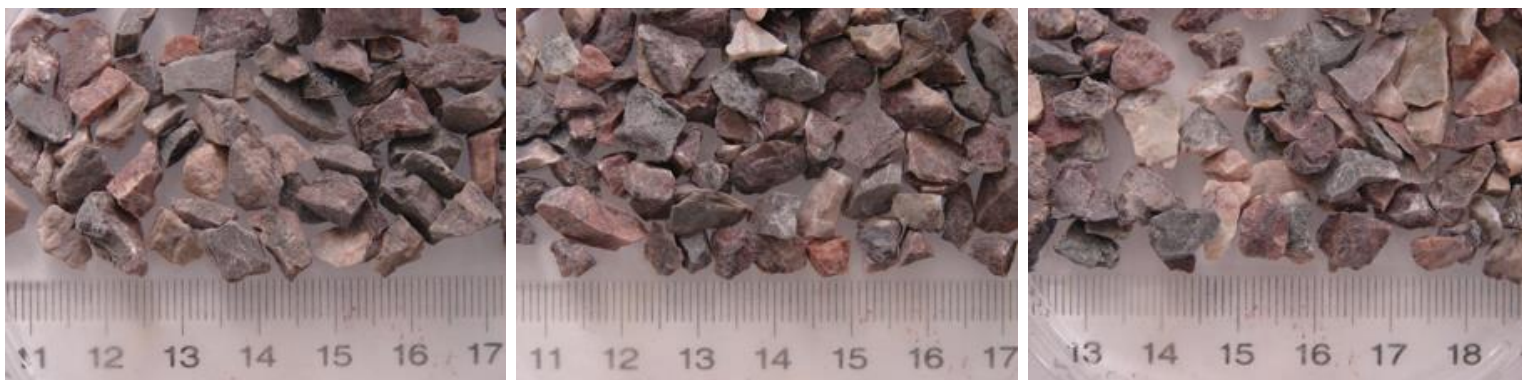

Figura 3. Amostra de minério silicatado de zinco de $6,3 \mathrm{~mm}$ a $4 \mathrm{~mm}$. Da esquerda para direita, alimentação, concentrado e rejeito.

Tabela 2. Caracterização química e mineralógica das amostras de Cobre

\begin{tabular}{|c|c|c|c|c|c|c|c|c|c|c|c|}
\hline \multirow{2}{*}{\multicolumn{2}{|c|}{ Amostra }} & \multirow{2}{*}{$\begin{array}{c}\text { Composição } \\
\text { mineralógica } \\
\text { (fases } \\
\text { predominantes) }\end{array}$} & \multirow{2}{*}{$\begin{array}{c}\text { Massa } \\
(\%)\end{array}$} & \multicolumn{8}{|c|}{ Concentração dos componentes (\%) } \\
\hline & & & & $\mathrm{Cu}$ & $\mathrm{SiO}_{2}$ & $\mathrm{Al}_{2} \mathrm{O}_{3}$ & $\mathrm{Fe}_{2} \mathrm{O}_{3}$ & $\mathrm{CaO}$ & $\mathrm{MgO}$ & $\mathrm{K}_{2} \mathrm{O}$ & $\mathrm{Na}_{2} \mathrm{O}$ \\
\hline \multirow{3}{*}{$\begin{array}{l}\text { Minério } \\
\text { Sulfetado } \\
\text { de Cobre }\end{array}$} & Alimentação & $\begin{array}{l}\text { Quartzo, Pirita, } \\
\text { Magnetita e } \\
\text { Calcopirita } \\
\end{array}$ & 100 & 0,75 & 52,6 & 8,36 & 15,9 & 2,19 & 2,11 & 3,42 & 1,02 \\
\hline & Concentrado & $\begin{array}{c}\text { Calcopirita, } \\
\text { Quartzo, Pirita e } \\
\text { Magnetita }\end{array}$ & 44 & 1,45 & 40,5 & 6,02 & 25,0 & 1,62 & 1,52 & 1,85 & 0,673 \\
\hline & Rejeito & $\begin{array}{c}\text { Quartzo, Albita, } \\
\text { Clorita, Microclínio } \\
\text { e Mica }\end{array}$ & 56 & 0,20 & 65,0 & 10,6 & 8,42 & 2,45 & 1,77 & 4,67 & 1,35 \\
\hline
\end{tabular}


Tabela 2. Caracterização química e mineralógica das amostras de Zinco

\begin{tabular}{|c|c|c|c|c|c|c|c|c|c|c|c|}
\hline \multirow{2}{*}{\multicolumn{2}{|c|}{ Amostra }} & \multirow{2}{*}{$\begin{array}{c}\text { Composição } \\
\text { mineralógica } \\
\text { (fases } \\
\text { predominantes) }\end{array}$} & \multirow{2}{*}{$\begin{array}{l}\text { Massa } \\
(\%)\end{array}$} & \multicolumn{8}{|c|}{ Concentração dos componentes (\%) } \\
\hline & & & & $\mathrm{Zn}$ & $\mathrm{SiO}_{2}$ & $\mathrm{Al}_{2} \mathrm{O}_{3}$ & $\mathrm{Fe}_{2} \mathrm{O}_{3}$ & $\mathrm{CaO}$ & $\mathrm{MgO}$ & $\mathrm{K}_{2} \mathrm{O}$ & $\mathrm{Na}_{2} \mathrm{O}$ \\
\hline \multirow{3}{*}{$\begin{array}{l}\text { Minério } \\
\text { Silicatado } \\
\text { de Zinco }\end{array}$} & Alimentação & $\begin{array}{c}\text { Willemita, Dolomita } \\
\text { e Hematita }\end{array}$ & 100 & 16,3 & 8,48 & 1,09 & 6,70 & 19,6 & 14,2 & 0,274 & 0,039 \\
\hline & Concentrado & $\begin{array}{c}\text { Willemita, Dolomita } \\
\text { e Hematita }\end{array}$ & 62,6 & 22,0 & 9,43 & 0,694 & 9,94 & 13,8 & 10,5 & 0,095 & 0,040 \\
\hline & Rejeito & $\begin{array}{c}\text { Dolomita, Willemita } \\
\text { e Hematita }\end{array}$ & 37,3 & 6,6 & 6,86 & 1,54 & 3,14 & 27,3 & 17,3 & 0,409 & 0,053 \\
\hline
\end{tabular}

Nota-se, na Tabela 2, que a pré-concentração do minério sulfetado de cobre gerou um concentrado com teor de $\mathrm{SiO}_{2}$ significativamente menor que a respectiva alimentação, ou seja, observa-se um enriquecimento em minerais portadores de SiO2, principalmente quartzo, no rejeito da pré-concentração. Ao contrário, o minério silicatado de zinco não exibiu grande variação no teor de SiO2 nos três fluxos analisados, provavelmente devido ao fato de que o silicato presente naquele minério seja o próprio mineral de minério $\left(\mathrm{Zn}_{2} \mathrm{SiO}_{4}\right)$, além de não ter sido detectada a presença de quartzo como mineral de ganga.

A Tabela 4 apresenta os resultados dos ensaios de abrasividade com a alimentação, concentrado e rejeito das amostras de minério silicatado de zinco e de cobre que passaram por um processo de pré-concentração por jigagem.

Tabela 4. Índice LCPC determinados para os minérios pré-concentrados

\begin{tabular}{|c|c|c|c|}
\hline \multicolumn{2}{|l|}{ Amostra } & \multirow{2}{*}{$\begin{array}{c}\text { Índice LCPC } \\
639 \\
625\end{array}$} & \multirow{2}{*}{$\begin{array}{c}\text { Média Índice LCPC } \\
632\end{array}$} \\
\hline \multirow{3}{*}{ Minério Silicatado de Zinco } & Alimentação & & \\
\hline & Concentrado & $\begin{array}{l}536 \\
677 \\
543 \\
\end{array}$ & 585 \\
\hline & Rejeito & $\begin{array}{l}460 \\
454 \\
439 \\
\end{array}$ & 450 \\
\hline \multirow{3}{*}{ Minério Sulfetado de Cobre } & Alimentação & $\begin{array}{l}1086 \\
1046 \\
1044\end{array}$ & 1059 \\
\hline & Concentrado & $\begin{array}{l}865 \\
692 \\
819 \\
752\end{array}$ & 782 \\
\hline & Rejeito & $\begin{array}{l}1124 \\
1100 \\
1092 \\
\end{array}$ & 1105 \\
\hline
\end{tabular}


Um dos aspectos que pode ser analisado por meio dos resultados apresentados nas Tabelas 2 e 3 é relação entre o teor de sílica na amostra e o valor obtido para sua abrasividade. Nota-se que os maiores valores do Índice LCPC foram obtidos nas amostras com maiores teores de sílica.

À exceção apenas da alimentação e do concentrado do minério silicatado de zinco, todos os outros resultados obtidos seguiram tal tendência, mostrando que o teor de sílica presente no minério tem grande influência sobre o valor obtido para a abrasividade deste.

Utilizando o Índice LCPC obtido para cada um dos fluxos (alimentação, concentrado e rejeito) do minério sulfetado de cobre, tem-se o gráfico mostrado na Figura 4.

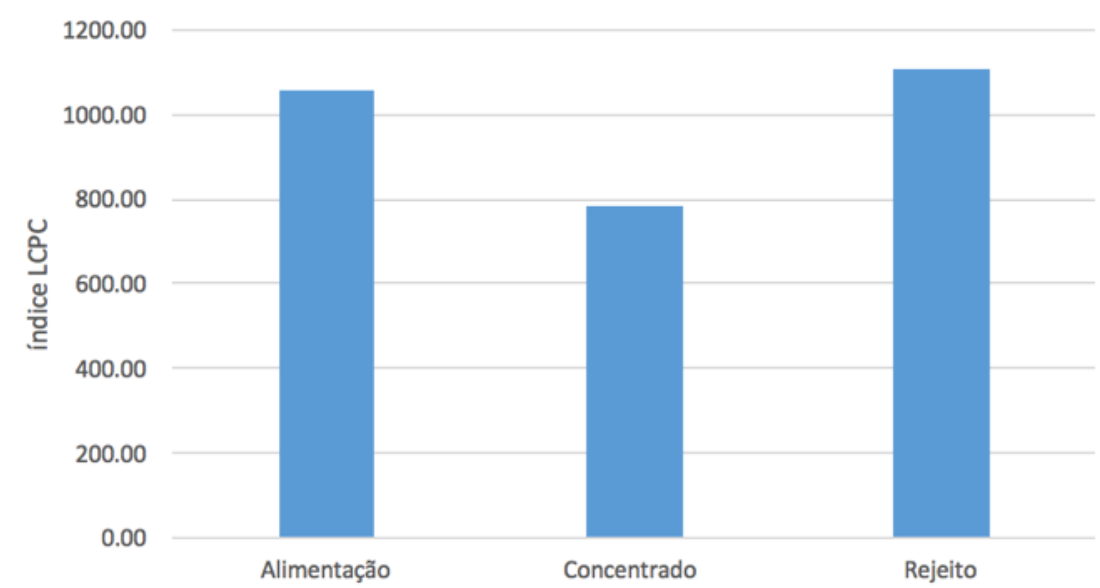

Figura 4. Comparação dos resultados LCPC para o minério sulfetado de cobre.

A partir dos resultados mostrados na Figura 4, pode-se concluir que neste caso, em termos de processamento mineral, seria vantajoso realizar a pré-concentração do ROM antes de alimentá-lo na planta de beneficiamento. Isso porque, como observado no gráfico, o produto concentrado apresentou uma abrasividade menor que a alimentação, o que resultaria em menor desgaste dos equipamentos de britagem e moagem e, consequentemente, menores custos na etapa de beneficiamento.

O segundo minério avaliado foi o minério silicatado de zinco e seus produtos da préconcentração por jigagem. Da mesma forma realizada para o minério sulfetado de cobre, o Índice LCPC obtido para cada um dos fluxos (alimentação, concentrado e rejeito) deste material são mostrados na Figura 5. 


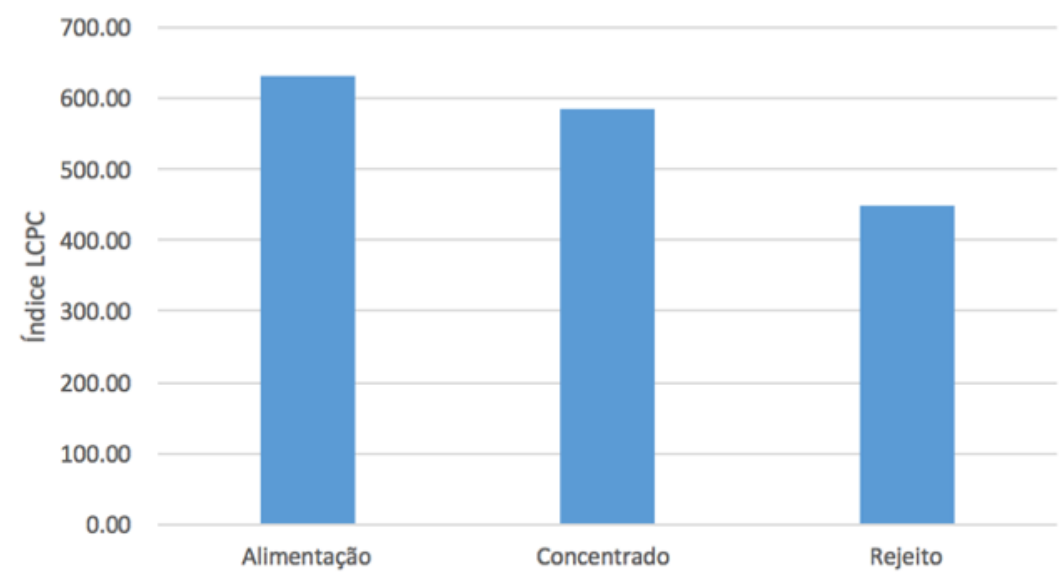

Figura 5. Comparação dos resultados LCPC para o minério silicatado de zinco.

Neste caso, nota-se que o processo de concentração por jigagem gerou materiais menos abrasivos do que a alimentação tanto no concentrado quanto no rejeito, resultado de certa forma inesperado, já que o correto seria haver um balanço entre a abrasividade dos três materiais. Por outro lado, a menor abrasividade do rejeito pode ser explicada pela sua composição mineralógica, já que este material é composto basicamente por carbonatos, que são pouco abrasivos. Este resultado também sugere que houve uma concentração de minerais abrasivos no concentrado, ao contrário do que seria esperado, já que normalmente tem-se uma maior concentração de minerais silicatados e mais abrasivos na ganga. Porém, por se tratar de um minério silicatado, neste caso, especificamente, é natural que haja a presença de sílica no material concentrado, uma vez que tal mineral está associado ao zinco, que é o mineral de interesse.

$\mathrm{Na}$ operação de uma usina, tal fato implicaria num maior desgaste por tonelada processada em relação à alimentação original, porém haveria uma massa muito menor sendo processada, já que uma grande parcela da ganga teria sido descartada, resultando então num desgaste total inferior quando comparado à uma situação em que a pré-concentração não fosse realizada.

Além dos valores obtidos para a abrasividade, também foi possível analisar visualmente a diferença de desgaste gerado nas plaquinhas por cada um dos materiais ensaiados.

No caso do minério sulfetado de cobre, na Figura 6, é possível notar nitidamente que a placa central, correspondente ao material pré-concentrado, está bem menos desgastada que as duas demais. Além disso, percebe-se que o desgaste maior ocorreu na terceira placa, que representa o material de rejeito, o mais abrasivo de acordo com os resultados numéricos obtidos. 


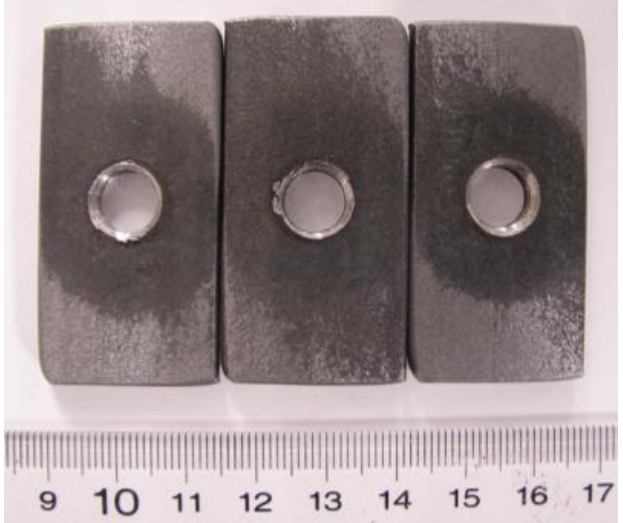

Figura 6. Plaquinhas utilizadas nos ensaios LCPC das amostras de minério sulfetado de cobre (da esquerda para a direita, alimentação, concentrado e rejeito).

Já no caso do minério silicatado de zinco, na Figura 7, as placas estão dispostas em ordem decrescente de abrasividade.

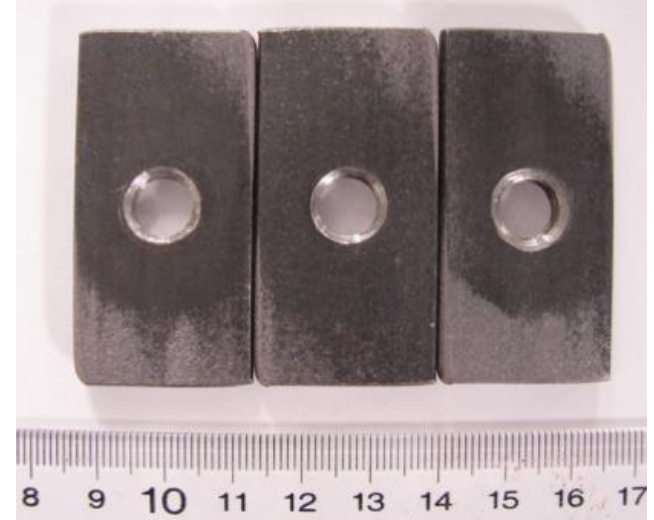

Figura 7. Plaquinhas utilizadas nos ensaios LCPC das amostras de minério silicatado de zinco (da esquerda para a direita, alimentação, concentrado e rejeito).

\section{CONCLUSÃo}

Apesar de serem necessários mais estudos para confirmar o que foi observado neste projeto, algumas tendências foram observadas. Verificou-se que, dependendo da composição mineralógica do minério, a pré-concentração realmente traz benefícios ao processo de beneficiamento industrial, por meio da redução da abrasividade do material que entra na planta, nos casos em que os silicatos se concentram nos minerais de ganga.

Notou-se, porém, que atenção especial é necessária quando a ganga é composta majoritariamente por minerais pouco abrasivos, como foi o caso do minério de minério silicatado de zinco estudado neste projeto. Neste caso, o processo da jigagem gerou uma concentração significativa de carbonatos no rejeito, o que resultou em um valor menor para a abrasividade desde produto. O concentrado, por sua vez, foi composto, basicamente, por materiais mais abrasivos, aumentando assim o valor de abrasividade deste material. Quando isto ocorre, é preciso ter ciência de que o processo de pré-concentração, apesar de já reduzir a massa que vai entrar na planta, também resultará no processamento de um material mais abrasivo do que o original, o que causaria um maior desgaste nos componentes por 
tonelada de minério processada. Entretanto, como a massa a ser alimentada seria significativamente menor, já que uma grande parcela da ganga foi descartada no processo de pré-concentração, o desgaste total dos equipamentos seria menor quando comparado à uma situação em que a pré-concentração não fosse realizada. Assim, mostra-se, mais uma vez, os benefícios da realização da etapa de préconcentração.

\section{Agradecimentos}

Agradecimentos especiais à Fundação do Instituto de Pesquisas Tecnológicas do Estado de São Paulo S/A, por financiar o projeto aqui apresentado através do edital $\mathrm{n}^{\circ}$ 08/2016.

Agradecemos também ao CNPq pelo apoio para a realização deste estudo, por meio do Projeto Universal processo 449932-2014-1

\section{REFERÊNCIAS}

1 BERGERMAN, M. G.; BARBOSA, F. A. M. ; TOMASELLI, B. Y. ; ROVERI, C. ; NAVARRO, F. C. . Pré-concentração de minerais sulfetados de zinco, chumbo e cobre utilizando-se beneficiamento gravimétrico. In: 67 Congresso internacional da ABM, 2012, Rio de Janeiro. Anais. São Paulo: ABM, 2012. v. 1. p. $1082-1092$.

2 SAMPAIO, J.A.; BALTAR, C.A.B.; SAVI, C.N.; CANCIAN, S.G. Fluorita: Nitro Química. In: SAMPAIO, J.A.; LUZ, A.B.; LINS, F.F. Usinas de beneficiamento de minérios no Brasil. Rio de Janeiro: CETEM/MCT, 2001. p. 51-60.

3 BERGERMAN, M.G. Ensaios em escala laboratorial para a determinação da susceptibilidade do minério de Aripuanã para a separação por meio denso. Poços de Caldas: Facepe, 2012a. 76 p. (Relatório Interno).

4 BERGERMAN, M.G. Ensaios em escala laboratorial para a determinação da susceptibilidade do minério de Santa Maria para a separação por meio denso. Poços de Caldas: Facepe, 2012b. 65 p. (Relatório Interno). BERGERMAN, M.G. Ensaios em escala laboratorial para a determinação da susceptibilidade do minério de Limoeiro para a separação por meio denso. Poços de Caldas: Facepe, 2012c. 45 p. (Relatório Interno).

6 BERGERMAN, M.G. Ensaios em escala laboratorial para a determinação da susceptibilidade do minério de Boa Esperança para a separação por meio denso. Poços de Caldas: Facepe, 2013a. 59 p. (Relatório Interno).

7 BERGERMAN, M.G. Ensaios em escala laboratorial para a determinação da susceptibilidade do minério de Anicuns para a separação por meio denso. Poços de Caldas: Facepe, 2013b. 59 p. (Relatório Interno).

8 BERGERMAN, M.G. Ensaios em escala laboratorial para a determinação da susceptibilidade do minério de Boa Esperança para a separação densitária. Poços de Caldas: Facepe, 2013c. 39 p. (Relatório Interno).

9 MASSOLA, C.P. Abrasão-corrosão em corpos moedores na moagem de minério de ferro. 2015. 106 p. Tese (Doutorado) - Escola Politécnica, Universidade de São Paulo, São Paulo, 2015.

10 BERGSTROM, B.H. Abrasiveness. In: WEISS, N.L. SME mineral processing handbook. New York: SME, 1985. v.2, p. 30-68/30-70.

11 Norme Française P18-579, 2013. Granulats - Détermination des coefficients d'abrasivité et de broyabilité.

12 KÄSLING, H.; THURO, K. Determining rock abrasivity in the laboratory. Proceedings of the European Rock Mechanics Symposium (EUROCK 2010), n. Figure 1, p. 1-4, 2010. 